phys. stat. sol. (b) 221, 425 (2000)

Subject classification: $78.47 .+\mathrm{p} ; \mathrm{S} 7.12$

\title{
100 fs Carrier Dynamics in GaAs under 100 nm Diameter Apertures
}

\author{
J. Hetzler ${ }^{1}$ ) (a), A. Brunner (a), M. Wegener (a), S. Leu (b), S. Nau (b), \\ and W. Stolz (b)
}

(a) Institut für Angewandte Physik, Universität Karlsruhe (TH), Kaiserstraße 12, D-76128 Karlsruhe, Germany

(b) Wiss. Zentrum für Materialwissenschaften (WZMW) und Fachbereich Physik, Philipps-Universität Marburg, Hans-Meerwein-Str., D-35032 Marburg, Germany

(Received April 10, 2000)

We study the decay of an optically excited cloud of carriers as a function of its initial diameter $d$. Values as low as $d=100 \mathrm{~nm}$ are possible via metallic apertures on bulk GaAs fabricated by electron beam lithography. We find that the decay time $\tau$ versus $d$ scales as $\tau \propto d^{2}$ for large $d$, as expected for diffusive transport, and as $\tau \propto d$ for submicron values of $d$, indicating strong deviations from diffusive transport. The dependence on parameters such as initial carrier kinetic energy, carrier density and sample temperature is discussed.

Consider a small cloud of carriers with transverse diameter $d$, which is impulsively excited by a short optical pulse, in a thin film of a semiconductor. It is clear that the cloud will spread out by carrier transport, thus the density in the initially excited area will decrease. This decay is not necessarily exponential, yet, for the sake of simplicity, we want to characterize it by a time constant $\tau$. How does $\tau$ depend on $d$ ? For large values of $d$, one obviously expects diffusive transport. In this case it is well known that $\tau$ is given by $\tau=\frac{1}{8 \ln 2} \frac{1}{D} d^{2} \propto d^{2}$ (initial Gaussian cloud with full width at half maximum $d$ ), with the diffusion constant $D$. Is this picture still correct on a scale of $d=100 \mathrm{~nm}$ ? For ballistic transport the carriers would obviously have some characteristic velocity $v$ and one would naively expect the dependence $\tau=d / v \propto d$. A similar expression holds for an expansion which is driven by the so-called Fermi pressure, which becomes relevant for (close to) degenerate carrier distributions.

In this paper, we experimentally study the dependence $\tau(d)$ in a thin film of bulk GaAs for values of $d$ ranging from several $\mu \mathrm{m}$ down to $100 \mathrm{~nm}$ at $T=20$ and $300 \mathrm{~K}$, for resonant and above-resonant excitation with $100 \mathrm{fs}$ pulses, and for carrier densities in the range of $10^{17}$ to $10^{18} \mathrm{~cm}^{-3}$. This extends and complements previous experiments [1 to 4$]$ as well as theoretical work [5,6] on this issue.

We employ a $100 \mathrm{~nm}$ thin film of bulk GaAs, grown by metal-organic vapor phase epitaxy. To avoid surface effects, the GaAs is clad between thin AlGaAs barriers. The layer structure is shown in Fig. 1 (except for two $5 \mathrm{~nm} \mathrm{GaAs}$ layers which protect the AlGaAs layers from oxidation). A $100 \mathrm{~nm}$ thick aluminum film is evaporated on top.

\footnotetext{
1) Corresponding author: Tel.: (++49) 721-608 3560; Fax: $(++49)$ 721-607 593; e-mail: jochen.hetzler@physik.uni-karlsruhe.de
} 


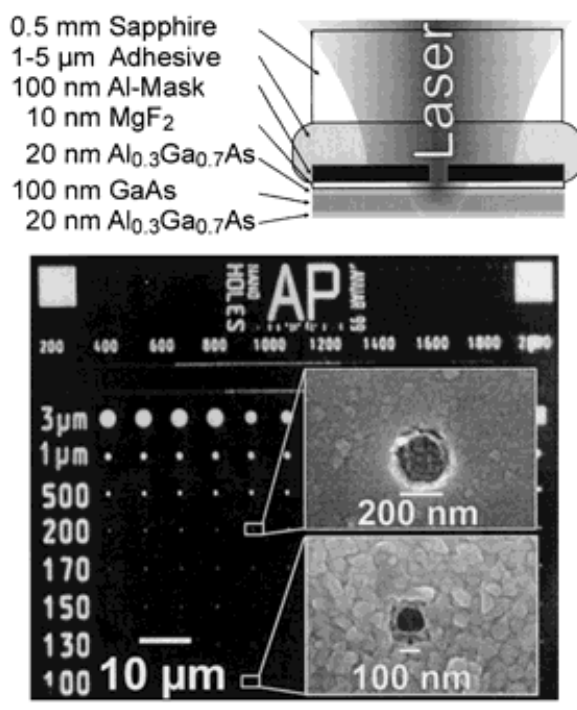

Fig. 1. Top: layer structure; bottom: microscope image of a typical set of nanoapertures (inset: electron micrographs of a 200 and a $100 \mathrm{~nm}$ aperture in the aluminum film)

Its measured optical density is five. To minimize interaction effects between the metal and the semiconductor, we introduce a $10 \mathrm{~nm}$ layer of isolating $\mathrm{MgF}_{2}$. Sets of defined apertures in a metal film [7] are fabricated with different diameter by standard electron-beam lithography with negative resist and subsequent lift-off. Figure 1 (bottom) shows a typical set of apertures under an optical microscope, the inset shows electron micrographs which reveal the quality of this process. Obviously, the GaAs film is within the optical near-field of these apertures. The samples are then glued to a sapphire disk and the GaAs substrate is etched-off to allow for transmission experiments. The $100 \mathrm{fs}$ laser pulses are focused to a diffraction limited spot size of $1.2 \mu \mathrm{m}$ by a microscope lens with a numerical aperture of NA $=0.4$. This allows to address single apertures in a pump-probe experiment. The pump intensity is ten times the probe intensity. Pump and probe propagate coaxially and have orthogonal circular polarization, which allows to isolate the probe from the pump on the detector. The carrier densities quoted in the following refer to that density which would be excited by the laser spot on a plane GaAs film (no aperture), assuming the unsaturated linear absorption coefficient.

We have performed pump-probe experiments at different excitation conditions through apertures from $100 \mathrm{~nm}$ to $1.5 \mu \mathrm{m}$. To complement these experiments, we have also done standard pump-probe experiments with focused laser spots with diameters between 1.2 and $8.8 \mu \mathrm{m}$ (no metal apertures) on the identical samples. The full width at half maximum $d$ of the Gaussian spots is carefully measured by scanning one of the metal apertures over the spot profile. The decay of the pump-probe traces shows a strong dependence on the aperture or spot diameter and on the excitation conditions. Eventhough the decays are usually non-exponential, we have fitted an exponential with time constant $\tau$ to the initial decay. The dependence of this initial decay time $\tau$ on the diameter $d$ for resonant and above-band-edge excitation at $T=20$ and $300 \mathrm{~K}$ is shown in Fig. 2.

To make sure that the observed decays are a result of the expansion of the small cloud of carriers, we perform the pump-probe experiments from the reverse side. The focused laser spot excites the GaAs film in front of the $\mathrm{Al}$ mask. The probe beam is detected through the aperture. Ideally, one would expect no influence of $d$ in this geometry. The small effects we have observed might be due to field enhancement at the edges of the apertures or due to small modifications of the potential landscape in the semiconductor film due to the presence of the metal aperture (strain or electrostatic). 


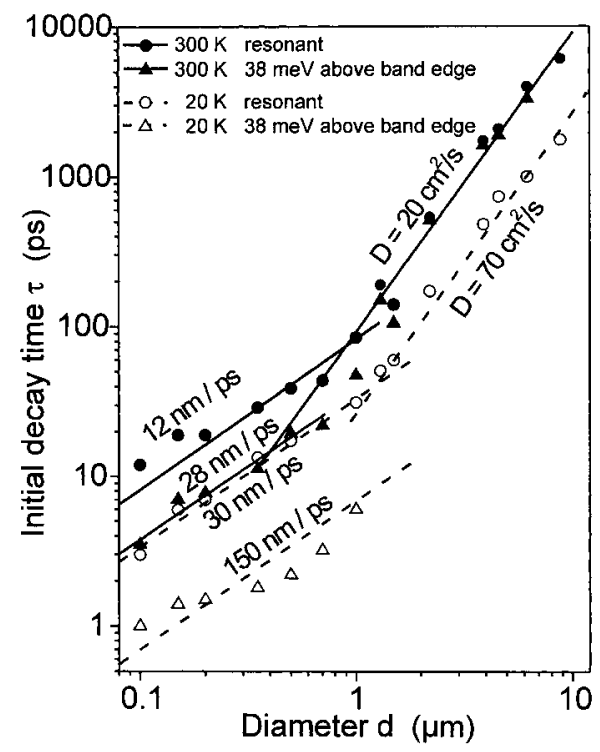

Fig. 2. Initial decay time $\tau$ of the pump-probe signal versus diameter $d$ of the excited area for resonant and above-resonant excitation at $T=20$ and $300 \mathrm{~K}$

Figure 2 shows a transition from the scaling according to $\tau \propto d^{2}$ for large $d$ to $\tau \propto d$ for submicron values of $d$, both for $T=20$ and $300 \mathrm{~K}$. This indicates a transition from diffusive transport to a different behavior with $\tau=d / v$. From the diffusive transport we can deduce a value of $D=70 \mathrm{~cm}^{2} / \mathrm{s}$ $(T=20 \mathrm{~K})$ and $D=20 \mathrm{~cm}^{2} / \mathrm{s}(T=300 \mathrm{~K})$, which is typical for GaAs structures [8]. In the introduction we have already stated that both, (i) a naive picture of ballistic transport, and (ii) expansion due to Fermi pressure, can lead to a dependence $\tau=d / v$. If the transport at small $d$ was ballistic for $T=20 \mathrm{~K}$ (dashed line) and $T=300 \mathrm{~K}$, (solid line) one would obviously expect no sample temperature dependence. Comparison of the solid and dashed lines shows that this is not the case. This indicates, that - at least for $T=300 \mathrm{~K}$ - transport is not simply ballistic. Yet, we do find a dependence $\tau=d / v$ for small $d$ even at $T=300 \mathrm{~K}$. This suggests that our results at small $d$ cannot be explained by ballistic transport alone. The difference between resonant and above-resonant $(38 \mathrm{meV})$ excitation, on the other hand, is exactly what one would expect for ballistic transport. This difference strongly suggests that carriers have not yet fully thermalized on the time scale of transport under the aperture. Transport driven by the so-called Fermi pressure also leads to an expansion with a characteristic velocity $v$. Thus, one would also expect a dependence according to $\tau=d / v$. In fact, the values for $v$ which have been measured [9] and computed [10] in the literature (for quasi-equilibrium carrier distributions) are in the same range as the ones that we determine from Fig. 2. An expansion driven by Fermi pressure should be density dependent, i.e. below a certain critical carrier density the Fermi pressure becomes insignificant. Unfortunately, in our experiment, we can only vary the optically excited carrier density $n_{\mathrm{eh}}$ between $10^{17}$ and $10^{18} \mathrm{~cm}^{-3}$. In this range, we have not found any significant influence of $n_{\mathrm{eh}}$ on the scenario shown in Fig. 2. However, these densities might still be too large to get below the critical density. We have not been able to perform pump-probe experiments with reasonable signal/noise ratios on single apertures with diameters as low as a few $100 \mathrm{~nm}$. The underlying difficulty becomes obvious when one calculates the total number of carriers below one aperture created by the pump. For a carrier density of $n_{\mathrm{eh}}=10^{17} \mathrm{~cm}^{-3}$, a $100 \mathrm{~nm}$ thin film, and a $100 \mathrm{~nm}$ diameter aperture one finds a total number of $10^{17} \mathrm{~cm}^{-3} \times 100 \mathrm{~nm} \times \pi(50 \mathrm{~nm})^{2}=78$ electron-hole pairs. Carrier densities of $n_{\mathrm{eh}}=10^{15} \mathrm{~cm}^{-3}$, which are usually considered as low, would correspond to less than one single electron under such a single aperture.

To summarize, we have performed $100 \mathrm{fs}$ pump-probe experiments on single apertures with diameters down to $100 \mathrm{~nm}$ on a $100 \mathrm{~nm}$ thin film of GaAs at $T=20$ and 
$300 \mathrm{~K}$. The measured dependences of the initial decay time $\tau$ versus the diameter of the initial carrier cloud $d$ at submicron values of $d$ show strong deviations from the scaling $\tau \propto d^{2}$, which would hold for diffusive transport. These deviations can neither be explained by a naive picture of ballistic transport nor by expansion driven by Fermi pressure (of equilibrium carrier distributions) alone. Possibly, our data can be explained by a combination of these two scenarios. We are currently not aware of any theory which has addressed transport of high-density plasmas in this regime of ultrasmall temporal and spatial scales.

Acknowledgements This work has been supported by the DFG through the SFB 195, the GRK 284 and the Leibniz-Preis 2000, and by the state Baden-Württemberg through the Schwerpunktprogramm "Nanotechnologie: Kontrolliert hergestellte Funktionselemente auf der Nanometerskala". It has been performed within the Institut für Nanotechnologie der Universität Karlsruhe. We acknowledge stimulating discussions with Th. Schimmel, S. W. Koch, and A. Knorr.

\section{References}

[1] S. Grosse, R. Arnold, G. v. Plessen, M. Кoch, J. Feldmann, V. M. Axt, T. Kuhn, R. Rettig, and W. Stolz, phys. stat. sol. (b) 204, 147 (1997).

[2] S. Smith, N. C. R. Holme, B. OrR, R. Kopelman, and T. Norris, Ultramicroscopy 71, 213 (1998).

[3] A. Richter, M. SÜPtitz, D. Heinrich, Ch. Lienau, T. Elsaesser, M. Ramsteiner, R. Nötzel, and K. H. Ploog, Appl. Phys. Lett. 73, 2176 (1998).

[4] M. Vollmer, H. Giessen, W. Stolz, W. W. Rühle, L. Ghislain, and V. Elings, Appl. Phys. Lett. 74, 1791 (1999).

[5] F. Steininger, A. Knorr, T. Stroucken, P. Thomas, and S. W. Косн, Phys. Rev. Lett. 77, 550 (1996).

[6] B. Hanewinkel, A. Knorr, P. Thomas, and S. W. Koch, Phys. Rev. B 60, 8975 (1999).

[7] D. Gammon, E. S. Snow, B. V. Shanabrook, D. S. Katzer, and D. Park, Phys. Rev. Lett. 76, 3005 (1996).

[8] H. Hillmer, S. Hansmann, A. Forchel, M. Morohashi, and E. Lopez, Appl. Phys. Lett. 53, 1937 (1988).

[9] K. M. RomaneK, H. Nather, J. Fischer, and E. O. Göbel, J. Lum. 24/25, 585 (1981)

[10] M. Сомbescot, Phys. Lett. A 85, 308 (1981). 